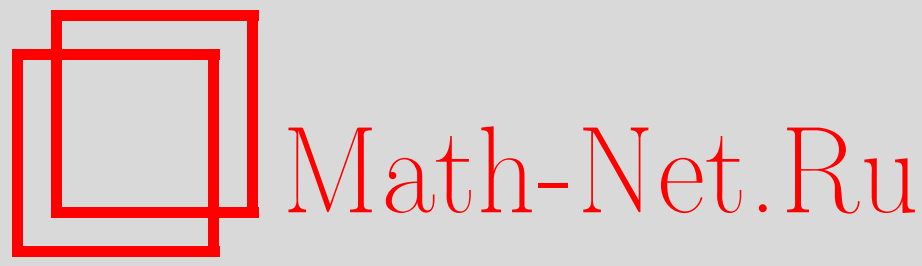

Х. Райбонгши, Н. Н. Сингх, Построение точно решаемых потенциалов в $D$-мерном уравнении Шредингера с массой, зависящей от координат, при помощи метода преобразований, ТМФ, 2015, том 183, номер 2, 312-328

DOI: https://doi.org/10.4213/tmf8784

Использование Общероссийского математического портала Math-Net.Ru подразумевает, что вы прочитали и согласны с пользовательским соглашением http://www . mathnet.ru/rus/agreement

Параметры загрузки:

IP: 34.227 .88 .159

26 апреля 2023 г., $17: 23: 36$

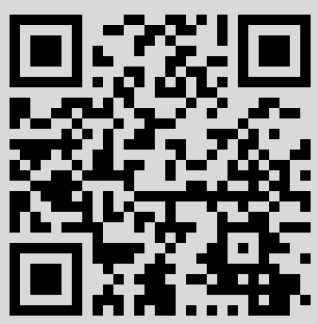




\section{ПОСТРОЕНИЕ ТОЧНО РЕШАЕМЫХ ПОТЕНЦИАЛОВ В D-МЕРНОМ УРАВНЕНИИ ШРЕДИНГЕРА С МАССОЙ, ЗАВИСЯЩЕЙ ОТ КООРДИНАТ, ПРИ ПОМОЩИ МЕТОДА ПРЕОБРАЗОВАНИЙ}

Метод обобщенных преобразований применяется к радиальному уравнению Шредингера с постоянной массой, которому удовлетворяет сферически-симметричный центральный потенциал, с целью получения точно решаемых квантовых систем с массой, зависящей от координат, в пространстве произвольной размерности в нерелятивистском пределе. Метод включает в себя координатное преобразование с последующим функциональным преобразованием и набор подстановок для функции массы, который приводит к появлению точно решаемых квантовых систем с массами, зависящими от координат. Также показано, что упорядочение Жу-Кремера для значений нефиксированных параметров оказывается естественным для систем с радиально-симметричными функциями масс и центральным потенциалом. В качестве примера метод применяется к потенциалу Меннинга-Розена и потенциалу Морса при различных выборах функций массы. Указано также на применение метода к потенциалу Халтена.

Ключевые слова: масса, зависящая от координат, точное аналитическое решение, потенциал Меннинга-Розена, потенциал Морса, обобщенное преобразование.

DOI: $10.4213 / \operatorname{tmf} 8784$

\section{1. ВВЕДЕНИЕ}

Точное аналитическое решение уравнения Шредингера для заданной квантовой системы сообщает максимально возможную информацию об этой системе. Однако имеется лишь весьма ограниченное число потенциалов, представляющих непосредственный физический интерес, которые допускают точные аналитические решения уравнения Шредингера. Поэтому для потенциалов, не являющихся точно решаемыми потенциалами (ТРП), часто приходится прибегать к различным методам приближений типа теории возмущений, к вариационному исчислению, приближению ВКБ

*Nalbari College, Nalbari, Assam, India. E-mail: hansrajb12345@gmail.com

${ }^{\dagger}$ Gauhati University, Guwahati, Assam, India. E-mail: nimai03@yahoo.com 
и т. д. Успешное применение таких приближенных методов к данной квантовой системе во многом зависит от того, насколько близок данный потенциал к какому-либо ТРП. В этой связи необходимо сконструировать по возможности максимальное количество ТРП, чтобы упростить квантово-механические вычисления. В духе подхода теории суперсимметрии (СУСИ) [1] был разработан метод преобразований, позволяющий получать новые точно решаемые квантовые системы из уже известных центральных ТРП фактически без решения соответствующего уравнения Шредингера. Этот метод известен как обобщенное преобразование (ОП) [2]. Многие авторы внесли значительный вклад в конструирование (и реконструирование) полиномиальных ТРП [3], неполиномиальных потенциалов [4]-[9] и потенциалов кольцевого типа [10] с применением метода ОП.

Из учебников физики твердого тела можно почерпнуть, что концепция эффективной массы, зависящей от координат, замечательным образом улучшает понимание целого ряда физических свойств, от электронной проводимости до сложных оптических явлений [11]. Огибающая функция зоны проводимости удовлетворяет уравнению Шредингера с массой, зависящей от координаты (МЗК-уравнению Шредингера). Так как масса является функцией координаты, она перестает коммутировать с оператором кинетической энергии, а оператор Гамильтона с МЗК становится неэрмитовым. Фон Роос предложил [12] (эрмитов) гамильтониан с МЗК, введя три подгоночных параметра, удовлетворяющих условию связи, в котором эффективный потенциал записывается в виде суммы функции профиля вещественного потенциала и модификации, возникающей из МЗК. Различные значения подгоночных параметров соответствуют различным гамильтонианам.

В отличие от обычного уравнения Шредингера, процедура решения МЗК-уравнений Шредингера оказывается более сложной. В последние годы значительные усилия были направлены на построение точного аналитического решения МЗК-уравнений Шредингера для разнообразных физически значимых квантово-механических потенциалов, таких как потенциал Халтена [13], потенциал Морса [14], потенциал Розена-Морса-Скарфа [15], потенциал с жестким кором [16], кулоновский потенциал [17], [18], гармонический потенциал [19], модифицированный потенциал Кратцеpa [20], сумма потенциала Халтена и потенциала гиперболического котангенса [21], потенциал типа гармонического осциллятора [22] и т. д., с применением различных методов, например метода Никифорова-Уварова [13], [14], [21], метода точечного канонического преобразования [23]-[25], метода деформированной алгебры [18], подхода СУСИ [26], [27], подхода квадратичных алгебр [28], метода сплетающих операторов [29], разложения решения в ряд [30], восстановления волновых пакетов [31], ћ-разложения [32], преобразования Дарбу [33], [34] и т. д. Эти исследования мотивировались плодотворными приложениями их результатов в различных областях науки о материалах и конденсированных состояниях материи. Например, можно найти применения этих методов в исследованиях электронных свойств полупроводников [35], кристаллов с градуированным составом [36], в исследованиях квантовых точек и квантовых потенциальных ям [37], [38], квантовых жидкостей [39], ядерных задачах многих тел [40] и т. д.

Стандартное ОП состоит в координатном преобразовании (КП) с последующим функциональным преобразованием $(Ф П)$ и наборе подходящих подстановок, которые являются неотъемлемой частью метода. КП - это основное преобразование, пе- 
реводящее одну систему в другую. ФП обеспечивает прямой путь построения волновых функций (в большинстве случаев нормируемых) для сконструированного ТРП из радиальных составляющих волновых функций исходного центрального ТРП.

Метод ОП, если он применяется к МЗК-уравнению Шредингера [41], [42], генерирует новую квантовую систему с новыми ТРП, собственными энергиями, точными собственными функциями и новой функцией массы. Функция массы новой системы при этом получается в результате преобразования функции массы исходной системы. В настоящей работе метод ОП применяется к радиальному уравнению Шредингера с постоянной массой, а трансформированное уравнение оказывается $D$ мерным МЗК-уравнением Шредингера; при этом необходимо выбрать подходящую функцию массы, приводящую к возникновению квантовой системы с новыми ТРП, функцией массы, собственными энергиями и точными собственными функциями энергии. Преобразованное уравнение показывает, что упорядочение ЖК-Кремера для параметров подгонки оказывается естественным в случае сферически-симметричных функций масс и центральных потенциалов. В методе используются потенциал Меннинга-Розена и потенциал Морса в качестве потенциалов исходной системы при различных выборах функций масс. Кроме того, указано, что можно применять этот метод в случае потенциала Халтена.

Структура статьи следующая. Метод генерации ТРП для $D$-мерных МЗК-уравнений Шредингера из уравнений Шредингера с постоянной массой и с известными центральными ТРП приведен в разделе 2. Применения метода построения ТРП с использованием потенциалов Меннинга-Розена и Морса при различных выборах функций масс содержатся в разделе 3, в котором также указана возможность применения нашего метода в случае потенциала Халтена. Заключение и список результатов приведены в завершающем разделе 4.

\section{2. ФОРМАЛИЗМ}

Эрмитов гамильтониан с МЗК имеет вид, предложенный фон Роосом [12]:

$$
H=-\frac{\hbar^{2}}{4}\left[M(r)^{\alpha} \vec{\nabla} M(r)^{\beta} \cdot \vec{\nabla} M(r)^{\gamma}+M(r)^{\gamma} \vec{\nabla} M(r)^{\beta} \cdot \vec{\nabla} M(r)^{\alpha}\right]+V(r) .
$$

Здесь параметры $\alpha, \beta, \gamma$ связаны соотношением $\alpha+\beta+\gamma=-1$, известным как неоднозначность фон Рооса, а $M(r)$ - МЗК. Значения параметров $\alpha, \beta, \gamma$ зависят от конкретной физической системы, например $\alpha=-1, \beta=\gamma=0$ (случай Горы-Вильяма), $\alpha=\gamma=0, \beta=-1$ (случай Бен Даниеля-Дюка), $\alpha=\gamma=-1 / 2, \beta=0$ (случай Жу-Кремера), $\beta=\gamma=-1 / 2, \alpha=0$ (случай Ли-Кюна), $\alpha=\gamma=-1 / 4, \beta=-1 / 2$ (случай Мустафы-Мажаримаусави). Все эти случаи суть различные упорядочения для значений параметров неоднозначности, которые можно найти в литературе [43]. На основе эрмитова гамильтониана (1) для сферически-симметричной функции массы $m(r)$ и потенциала $V(r)$ можно записать $D$-мерное стационарное МЗК-уравнение Шредингера в виде [44]

$$
\left(-\vec{\nabla}_{D} \frac{1}{m(r)} \cdot \vec{\nabla}_{D}+V^{\mathrm{eff}}(r)\right) \chi(\vec{r})=E \chi(\vec{r}),
$$


где $M(r)=m_{0} m(r)$, а $m_{0}$ и $m(r)$ - постоянная масса и безразмерная функция массы; $\hbar=1=2 m_{0}$. Эффективный потенциал $V^{\mathrm{eff}}(r)$ задается выражением

$$
V^{\mathrm{eff}}(r)=V(r)+U_{\alpha \beta}(r)
$$

где

$$
U_{\alpha \beta}(r)=\frac{1}{2}(\beta+1) \frac{m^{\prime \prime}(r)}{m^{2}(r)}-[\alpha(\alpha+\beta+1)+\beta+1] \frac{\left(m^{\prime}\right)^{2}(r)}{m^{3}(r)}
$$

представляет собой модификацию потенциала, зависящую от функции массы $m(r)$ и от параметров неоднозначности $\alpha, \beta, \gamma$. Штрихом обозначается дифференцирование функции по ее аргументу. $D$-мерная волновая функция сферически-симметричного уравнения Шредингера имеет вид

$$
\chi(\vec{r})=r^{-(D-1) / 2} R_{n_{r}, l_{D}}(r) Y_{l_{D}, m_{D}}\left(\theta_{1}, \theta_{2}, \ldots, \theta_{D-2}, \phi\right) .
$$

Применяя это соотношение к уравнению (2), получим следующее $D$-мерное радиальное МЗК-уравнение Шредингера [44]:

$$
\left[\frac{d^{2}}{d r^{2}}+\frac{m^{\prime}(r)}{m(r)}\left(\frac{D-1}{2 r}-\frac{d}{d r}\right)-\frac{l_{D}\left(l_{D}+1\right)}{r^{2}}+m(r)\left(E_{n}-V^{\mathrm{eff}}(r)\right)\right] R_{n_{r}, l_{D}}(r)=0
$$

где $l_{D}=l+(D-3) / 2$ при $D \geqslant 2, l$ - регулярное квантовое число углового момента, а $n_{r}=0,1,2, \ldots$ - радиальное квантовое число. Полагая в (4), что радиальная волновая функция имеет вид $\psi(r)=r^{-(D-1) / 2} R_{n_{r}, l_{D}}(r)$, получаем следующее уравнение:

$$
\begin{aligned}
\psi^{\prime \prime}(r) & -\left(\frac{m^{\prime}(r)}{m(r)}-\frac{D-1}{r}\right) \psi^{\prime}(r)+ \\
& +m(r)\left(E_{n}-V^{\mathrm{eff}}(r)-\frac{l(l+D-2)}{r^{2}} \frac{1}{m(r)}\right) \psi(r)=0 .
\end{aligned}
$$

Это радиальное МЗК-уравнение Шредингера в произвольном $D$-мерном евклидовом пространстве для сферически-симметричной функции массы $m(r)$ и центрального потенциала $V(r)$.

С другой стороны, радиальное уравнение Шредингера для системы с постоянной массой в трехмерном евклидовом пространстве (системы $A$ ) имеет вид

$$
\psi_{A}^{\prime \prime}(r)+\frac{2}{r} \psi_{A}^{\prime}(r)+\left(E_{n}-V_{A}(r)-\frac{l(l+1)}{r^{2}}\right) \psi_{A}(r)=0
$$

где $V_{A}(r)$ - сферически-симметричный центральный ТРП. Будем считать $V_{A}(r)$ известным неполиномиальным потенциалом. В отличие от полиномиальных потенциалов, точные решения для неполиномиальных потенциалов известны только для случая s-волн. Для s-волны $(l=0)$ уравнение $(6)$ принимает вид

$$
\psi_{A}^{\prime \prime}(r)+\frac{2}{r} \psi_{A}^{\prime}(r)+\left(E_{n}^{A}-V_{A}(r)\right) \psi_{A}(r)=0
$$

где собственные энергии $E_{n}^{A}$ и нормированные собственные функции $\psi_{A}(r)$ известны при заданном $V_{A}(r)$. 
Наша цель состоит в том, чтобы преобразовать уравнение (7) к виду (5) (система $B)$, применив метод ОП, включаюший в себя КП

$$
r \mapsto g_{B}(r)
$$

и далее $Ф П$

$$
\psi_{B}(r)=f_{B}^{-1}(r) \psi_{A}\left(g_{B}(r)\right) .
$$

Применяя формулы (8), (9) к уравнению (7), получаем

$$
\begin{aligned}
\psi_{B}^{\prime \prime}(r) & +\left(\frac{d}{d r} \ln \frac{f_{B}^{2} g_{B}^{2}}{g_{B}^{\prime}}\right) \psi_{B}^{\prime}(r)+ \\
& +\left[\left(\frac{d}{d r} \ln f_{B}\right)\left(\frac{d}{d r} \ln \frac{f_{B}^{\prime} g_{B}^{2}}{g_{B}^{\prime}}\right)+\left(g_{B}^{\prime}\right)^{2}\left(E_{n}^{A}-V_{A}\left(g_{B}\right)\right)\right] \psi_{B}(r)=0 .
\end{aligned}
$$

Функция $f_{B}^{-1}(r)$ в этом выражении представляет собой модулированную амплитуду, а функция преобразования $g_{B}(r)$ должна быть минимум трижды дифференцируемой. Из условия согласования следует, что коэффициент при $\psi_{B}^{\prime}(r)$ в уравнении $(10)$ должен быть равен $-m^{\prime}(r) / m(r)+(D-1) / r$. Поэтому положим

$$
\frac{d}{d r} \ln \frac{f_{B}^{2} g_{B}^{2}}{g_{B}^{\prime}}=-\left(\frac{m^{\prime}(r)}{m(r)}-\frac{D-1}{r}\right),
$$

что позволяет представить $f_{B}(r)$ как функцию от $g_{B}(r), g_{B}^{\prime}(r)$ и функции массы $m(r)$ :

$$
f_{B}(r)=r^{(D-1) / 2} g_{B}^{-1} m^{1 / 2}\left(g_{B}^{\prime}\right)^{1 / 2} .
$$

Подставив уравнение (11) в (10), получим

$$
\begin{aligned}
\psi_{B}^{\prime \prime}(r) & -\left(\frac{m^{\prime}}{m}-\frac{D-1}{r}\right) \psi_{B}^{\prime}(r)+ \\
& +\left[\frac{1}{2}\left\{g_{B}, r\right\}-\frac{1}{2}\left(\frac{m^{\prime \prime}}{m}-\frac{3}{2}\left(\frac{m^{\prime}}{m}\right)^{2}\right)-\left(\frac{D-1}{2}\right) \frac{m^{\prime}}{m} \frac{1}{r}+\right. \\
& \left.+\left(\frac{D-1}{2}\right)\left(\frac{D-3}{2}\right) \frac{1}{r^{2}}+\left(g_{B}^{\prime}\right)^{2}\left(E_{n}^{A}-V_{A}\left(g_{B}\right)\right)\right] \psi_{B}(r)=0,
\end{aligned}
$$

где

$$
\left\{g_{B}, r\right\}=\frac{g_{B}^{\prime \prime \prime}}{g_{B}^{\prime}}-\frac{3}{2}\left(\frac{g_{B}^{\prime \prime}}{g_{B}^{\prime}}\right)^{2}
$$

обозначает производную Шварца.

Если порождающая система задается потенциалом в виде многочлена с $n$ слагаемыми, то имеются $2^{n}-1$ различных способов выбрать различные члены в качестве рабочего потенциала. Для математической простоты будем считать, что в качестве рабочего потенциала выбирается единственный член, который обозначается как $V_{A}^{\mathrm{w}}(r)$. Чтобы записать уравнение (12) в стандартном виде $D$-мерного радиального МЗК-уравнения Шредингера, можно выбрать следующий подходящий анзац, 
представляющий собой неотъемлемую часть метода преобразований:

$$
\begin{gathered}
\frac{\left(g_{B}^{\prime}\right)^{2}}{m} V_{A}^{\mathrm{w}}\left(g_{B}\right)=-E_{n}^{B}, \\
\frac{\left(g_{B}^{\prime}\right)^{2}}{m} E_{n}^{A}=-V_{B}^{(1)}(r), \\
\frac{\left(g_{B}^{\prime}\right)^{2}}{m}\left(V_{A}\left(g_{B}\right)-V_{A}^{\mathrm{w}}\left(g_{B}\right)\right)=V_{B}^{(2)}(r), \\
\frac{1}{m}\left(\frac{1}{2}\left\{g_{B}, r\right\}+\left(\frac{D-1}{2}\right)\left(\frac{D-3}{2}\right) \frac{1}{r^{2}}\right)=-V_{B}^{(3)}(r)
\end{gathered}
$$

и

$$
U_{\alpha \beta}(r)=\frac{1}{2 m}\left(\frac{m^{\prime \prime}}{m}-\frac{3}{2}\left(\frac{m^{\prime}}{m}\right)^{2}\right) .
$$

В формуле $(13) V_{A}^{\mathrm{w}}\left(g_{B}\right)$ есть заданная функция от $g_{B}(r)$, в то время как $g_{B}(r)$ и $m(r)$ остаются неизвестными. Выбирая $m(r)$ в виде функции от $g_{B}(r)$, получаем, что функция массы $m(r)$ становится зависящей от координаты и объединяется с потенциалом, и это приводит к зависимости взаимодействия от массы. Поэтому положим $m(r)=k f\left(g_{B}(r)\right)$, где $k=$ const. Тогда равенство (13) принимает вид

$$
\frac{\left(g_{B}^{\prime}\right)^{2}}{f\left(g_{B}(r)\right)} V_{A}^{\mathrm{w}}\left(g_{B}\right)=-k E_{n}^{B} .
$$

На этом этапе следует тщательно выбирать функцию массы $m(r)$ как функцию от $g_{B}(r)$, чтобы обеспечить интегрируемость квадратного корня из выражения (18) для хорошо себя ведущей функции $g_{B}(r)$. Из формулы (18) следует ограничение на число ТРП (с МЗК), порождающихся определенным центральным ТРП. В реальности функция $g_{B}(r)$ вычисляется путем решения уравнения (18) при различных выборах функции массы. Как только найдена функция $g_{B}(r)$, становится легче вычислять функции $V_{B}^{(1)}(r), V_{B}^{(2)}(r)$ и $V_{B}^{(3)}(r)$ с помощью уравнений $(14)-(16)$, тем самым мы получаем потенциал системы $B$ (с MЗK) в $D$-мерном евклидовом пространстве:

$$
V_{B}(r)=V_{B}^{(1)}(r)+V_{B}^{(2)}(r)+V_{B}^{(3)}(r) .
$$

Величина

$$
\left(\frac{D-1}{2}\right)\left(\frac{D-3}{2}\right) \frac{1}{r^{2}}
$$

в выражении для потенциала (16) приводит к появлению фонового потенциала притяжения, который зависит от обратного квадрата расстояния в любой размерности, кроме случаев размерностей 1 и 3 , где порождаемый потенциал имеет чисто неполиномиальный вид. В случае потенциалов полиномиального типа этот фоновый потенциал в совокупности с потенциалом, задающимся производной Шварца, т. е.

$$
\frac{1}{2}\left\{g_{B}, r\right\}+\left(\frac{D-1}{2}\right)\left(\frac{D-3}{2}\right) \frac{1}{r^{2}}
$$

в формуле (16), порождает правильный центробежный потенциал барьерного типа в произвольной размерности [2]. 
Интересно отметить, что потенциал $U_{\alpha \beta}(r)$, заданный в $(17)$, имеет тот же вид, что и модификация потенциала из формулы (3). Сравнивая соотношения (17) и (3), получаем $\alpha=\gamma=-1 / 2, \beta=0$, что совпадает с упорядочением Жу-Кремера для параметров неоднозначности. Поэтому для сферически-симметричной функции массы и центрального потенциала упорядочение Жу-Кремера параметров неоднозначности оказывается естественным.

Наконец, из уравнения (12) получаем радиальное МЗК-уравнение Шредингера в $D$-мерном евклидовом пространстве для системы $B$ :

$$
\psi_{B}^{\prime \prime}(r)-\left(\frac{m^{\prime}(r)}{m(r)}-\frac{D-1}{r}\right) \psi_{B}^{\prime}(r)+m(r)\left(E_{n}^{B}-V_{B}^{\mathrm{eff}}(r)\right) \psi_{B}(r)=0
$$

где

$$
V_{B}^{\mathrm{eff}}(r)=V_{B}(r)+U_{\alpha \beta}(r)+\left(\frac{D-1}{2}\right) \frac{m^{\prime}(r)}{m^{2}(r)} \frac{1}{r} .
$$

Собственные функции системы $B$ получаются из соотношений $(9),(11)$ и имеют вид

$$
\psi_{B}(r)=r^{-(D-1) / 2} g_{B} m^{1 / 2}\left(g_{B}^{\prime}\right)^{-1 / 2} \psi_{A}\left(g_{B}\right)
$$

Эти функции известны, поскольку известны функции $\psi_{A}(r), g_{B}(r)$ и $m(r)$.

В (14) не зависящий от $r$ множитель можно записать [2] как $f\left(E_{n}^{B}\right) E_{n}^{A}=C_{B}^{2}$, где $C_{B}^{2}$ - характеристическая постоянная системы $B$. Тем самым можно получить собственные энергии системы $B$ :

$$
E_{n}^{B}=f^{-1}\left(\frac{C_{B}^{2}}{E_{n}^{A}}\right)
$$

Эти значения известны, поскольку известны величины $E_{n}^{A}$.

Одним из наиболее важных свойств волновых функций построенной системы $B$ оказывается то, что они нормируемы в большинстве случаев. Из формул (20) и (13) получается условие нормировки для $\psi_{B}(r)$ в виде [42]

$$
\left|N_{B}\right|^{2} \int_{g_{B}(0)}^{g_{B}(\propto)} \psi_{A}^{*}(r)\left(-\frac{V_{A}^{\mathrm{w}}(r)}{E_{n}^{B}}\right) \psi_{A}(r) r^{D-1} d r=1 \text {. }
$$

Поэтому нормируемы все те функции $\psi_{B}(r)$, для которых $E_{n}^{B} \neq 0$, а интеграл

$$
I\left[g_{B}(\propto), g_{B}(0)\right]=\int_{g_{B}(0)}^{g_{B}(\propto)} \psi_{A}^{*}(r)\left(-\frac{V_{A}^{\mathrm{w}}(r)}{E_{n}^{B}}\right) \psi_{A}(r) r^{D-1} d r
$$

конечен. Так как $\psi_{A}(r)$ представляет собой нормированную волновую функцию физической системы, а $V_{A}^{\mathrm{w}}$ - часть точно решаемого потенциала, величина

$$
-E_{n}^{B} I\left[g_{B}(\propto), g_{B}(0)\right]=\left\langle V_{A}^{\mathrm{w}}(r)\right\rangle
$$

существует, и преобразованные волновые функции $\psi_{B}(r)$ нормируемы. 


\section{3. ПРИЛОЖЕНИЯ}

В качестве приложений метода рассмотрим ТРП Меннинга-Розена и Морса в качестве примера порождающих новые ТРП физических квантовых систем.

\section{1. Генерация ТРП с использованием потенциала Меннинга-Розена.} ТРП Меннинга-Розена задается выражением [7]

$$
V_{A}(r)=\frac{1}{b^{2}}\left(\frac{\epsilon(\epsilon-1) e^{-2 r / b}}{\left(1-e^{-r / b}\right)^{2}}-\frac{A e^{-r / b}}{1-e^{-r / b}}\right),
$$

в котором $A$ и $b$ - параметры. Собственные энергии и радиальные волновые функции имеют вид

$$
\begin{aligned}
E_{n}^{A} & =-\frac{1}{b^{2}}\left(\frac{A-\epsilon}{2(\epsilon+n)}-\frac{n(n+2 \epsilon)}{2(\epsilon+n)}\right)^{2}, \\
\psi_{A}(r) & =N_{A} r^{-1}\left(1-e^{-r / b}\right)^{\epsilon} e^{-\lambda_{A} r / b}{ }_{2} F_{1}\left(-n, 2\left(\epsilon+\lambda_{A}\right)+n ; 1+2 \lambda_{A} ; e^{-r / b}\right) .
\end{aligned}
$$

Здесь $N_{A}$ - нормировочная константа, $\lambda_{A}=\sqrt{b^{2}\left(-E_{n}^{A}\right)}$,

$$
\begin{gathered}
\epsilon+\lambda_{A}-\sqrt{A+\epsilon(\epsilon-1)+\lambda_{A}^{2}}=-n, \quad n=1,2, \ldots, n_{\max } \\
n_{\max }=\sqrt{A+\epsilon(\epsilon-1)}-\epsilon
\end{gathered}
$$

и ${ }_{2} F_{1}\left(-n, 2\left(\epsilon+\lambda_{A}\right)+n ; 1+2 \lambda_{A} ; e^{-r / b}\right)-$ гипергеометрическая функция.

Выберем сначала следующий рабочий потенциал из (21):

$$
V_{A}^{\mathrm{w}}(r)=-\frac{A e^{-r / b}}{b^{2}\left(1-e^{-r / b}\right)} .
$$

Рассмотрим несколько вариантов выбора функции массы (для $k=1$ ).

3.1.1. Случай $m(r)=e^{-g_{B} / b}$. Применяя соотношения $(24)$ и (13), получаем функцию преобразования

$$
g_{B}(r)=b \ln \operatorname{ch}^{2} \eta_{n} r
$$

которая дает локальное свойство $g_{B}(0)=0$ и функцию массы

$$
m(r)=\operatorname{sech}^{2} \eta_{n} r
$$

где

$$
\eta_{n}^{2}=\frac{E_{n}^{B}}{4 A}
$$

Подставляя (25) и (26) в формулы (14)-(17), получим соответственно

$$
\begin{aligned}
V_{B}^{(1)}(r) & =C_{B}^{2} \operatorname{sh}^{2} \eta_{n} r \\
V_{B}^{(2)}(r) & =4 \eta_{n}^{2} \epsilon(\epsilon-1) \operatorname{csch}^{2} \eta_{n} r \\
V_{B}^{(3)}(r) & =\frac{\eta_{n}^{2}}{2}\left(1+\operatorname{cth}^{2} \eta_{n} r\right)+\frac{\eta_{n}^{2}}{4} \operatorname{csch}^{2} \eta_{n} r-\frac{(D-1)(D-3)}{4 r^{2}} \frac{1}{m(r)}
\end{aligned}
$$


и

$$
U_{\alpha \beta}(r)=-\eta_{n}^{2} \operatorname{ch}^{2} \eta_{n} r
$$

Здесь

$$
C_{B}^{2}=-4 b^{2} \eta_{n}^{2} E_{n}^{A}
$$

есть характеристическая постоянная системы $B$. И окончательно, потенциал системы $B(19)$ принимает вид

$$
\begin{aligned}
V_{B}(r)= & C_{B}^{2} \operatorname{sh}^{2} \eta_{n} r+\eta_{n}^{2}\left(4 \epsilon(\epsilon-1)+\frac{1}{4}\right) \operatorname{csch}^{2} \eta_{n} r+ \\
& +\frac{\eta_{n}^{2}}{2}\left(1+\operatorname{cth}^{2} \eta_{n} r\right)-\frac{(D-1)(D-3)}{4 r^{2}} \frac{1}{m(r)} .
\end{aligned}
$$

Потенциал $V_{B}(r)$ представляет собой штурмиан, который зависит от энергии, т. е. от квантового числа $n$, так как $\eta_{n}$ зависит от $n$. Каждое значение $n$ задает квантовую систему, и система $B$ поэтому состоит из более чем одной квантовой системы. Чтобы привести систему $B$ к нормальному виду, проведем масштабирование $A \rightarrow A_{n}=E_{n}^{B} / 4 s^{2}$, где $s-$ масштабный множитель. Это делает величину $\eta_{n}^{2}=s^{2}$ постоянной. Далее, из равенств (27) и (28) получим собственные энергии системы $B$

$$
E_{n}^{B}=4 s^{2}\left[n^{2}+2\left(\epsilon \lambda_{B}+\epsilon n+\lambda_{B} n\right)+\epsilon\right],
$$

потенциал в нормальном виде

$$
\begin{aligned}
V_{B}(r)= & C_{B}^{2} \operatorname{sh}^{2} s r+s^{2}\left(4 \epsilon(\epsilon-1)+\frac{1}{4}\right) \operatorname{csch}^{2} s r+ \\
& +\frac{s^{2}}{2}\left(1+\operatorname{cth}^{2} s r\right)-\frac{(D-1)(D-3)}{4 r^{2}} \frac{1}{m(r)}
\end{aligned}
$$

и модифицированный потенциал

$$
U_{\alpha \beta}(r)=-s^{2} \operatorname{ch}^{2} s r .
$$

Точные собственные функции системы $B$ получаются из соотношений (20) и (23):

$$
\begin{aligned}
\psi_{B}(r)= & N_{B} r^{-(D-1) / 2} \operatorname{th}^{2 \epsilon-1 / 2} s r \operatorname{sech}^{2 \lambda_{B}+1} s r \times \\
& \times{ }_{2} F_{1}\left(-n, 2\left(\epsilon+\lambda_{B}\right)+n ; 1+2 \lambda_{B} ; \operatorname{sech}^{2} s r\right),
\end{aligned}
$$

где $\lambda_{B}=C_{B} / 2 s, N_{B}-$ нормировочный множитель и

$$
2 s \epsilon+C_{B}-\sqrt{E_{n}^{B}+4 s^{2} \epsilon(\epsilon-1)+C_{B}^{2}}=-2 s n
$$

(см. рис. 1). На рис. 1-3 параметры $s=1, C_{B}=1,2,1, \epsilon=0.95,0.95,1.95$, а $D=3$.

3.1.2. Случай $m(r)=e^{g_{B} / b}$. Применяя ту же процедуру, что выше, получим функцию преобразования

$$
g_{B}(r)=b \ln \frac{1}{1-X^{2}(r)},
$$

функцию массы

$$
m(r)=\frac{1}{1-X^{2}(r)},
$$




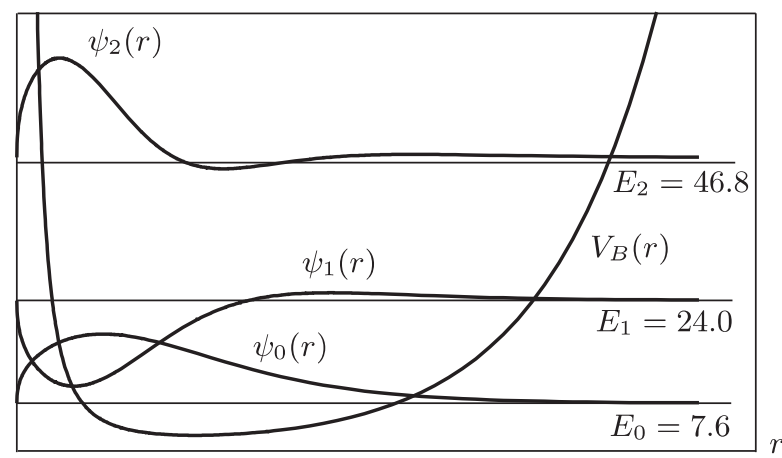

Рис. 1. ТРП в случае $m(r)=e^{-g_{B} / b}$ и отвечающие ему собственные значения и собственные функции для $n=0,1,2$.

потенциал в нормальном виде

$$
\begin{aligned}
V_{B}(r)= & \left(C_{B}^{2}-s^{2}\right)\left(\frac{1}{X^{2}(r)}-1\right)^{-1}+ \\
& +s^{2}\left(4 \epsilon(\epsilon-1)+\frac{3}{4}\right)\left(\frac{1}{X^{2}(r)}-1\right)-\frac{(D-1)(D-3)}{4 r^{2}} \frac{1}{m(r)},
\end{aligned}
$$

модифицированный потенциал

$$
U_{\alpha \beta}(r)=-\frac{s^{2}}{X^{2}(r)-1},
$$

собственные энергии и нормируемые собственные функции

$$
\begin{aligned}
E_{n}^{B}= & 4 s^{2}\left(n^{2}+2\left(\epsilon \lambda_{B}+\epsilon n+\lambda_{B} n\right)+\epsilon\right), \\
\psi_{B}(r)= & N_{B} r^{-(D-1) / 2} X^{2 \epsilon-1 / 2}(r)\left(1-X^{2}(r)\right)^{\lambda_{B}} \times \\
& \times{ }_{2} F_{1}\left(-n, 2\left(\epsilon+\lambda_{B}\right)+n ; 1+2 \lambda_{B} ;\left(1-X^{2}(r)\right)\right)
\end{aligned}
$$

для системы $B$, где $N_{B}$ - нормировочный множитель, $X(r)=s r, \lambda_{B}=C_{B} / 2 s$,

$$
2 s \epsilon+C_{B}-\sqrt{E_{n}^{B}+4 s^{2} \epsilon(\epsilon-1)+C_{B}^{2}}=-2 s n,
$$

а $C_{B}^{2}$ - характеристическая постоянная (см. рис. 2).

3.1.3. Случай $m(r)=\left(1-e^{-g_{B} / b}\right)^{-1}$. Этот вариант выбора $m(r)$ дает функцию преобразования

$$
g_{B}(r)=-2 b \ln y(r)
$$

функцию массы

потенциал в нормальном виде

$$
m(r)=\frac{1}{1-y^{2}(r)},
$$

$$
\begin{aligned}
V_{B}(r)= & \left(C_{B}^{2}-\frac{s^{2}}{4}\right)\left(\frac{1}{y^{2}(r)-1}\right)+ \\
& +4 s^{2} \epsilon(\epsilon-1)\left(\frac{1}{y^{2}(r)-1}\right)^{-1}-\frac{(D-1)(D-3)}{4 r^{2}} \frac{1}{m(r)},
\end{aligned}
$$




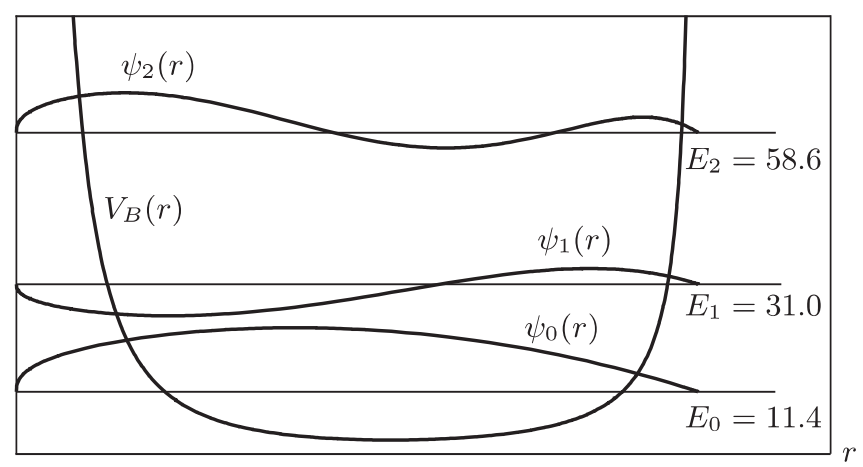

Рис. 2. ТРП в случае $m(r)=e^{g_{B} / b}$ и отвечающие ему собственные значения и собственные функции для $n=0,1,2$.

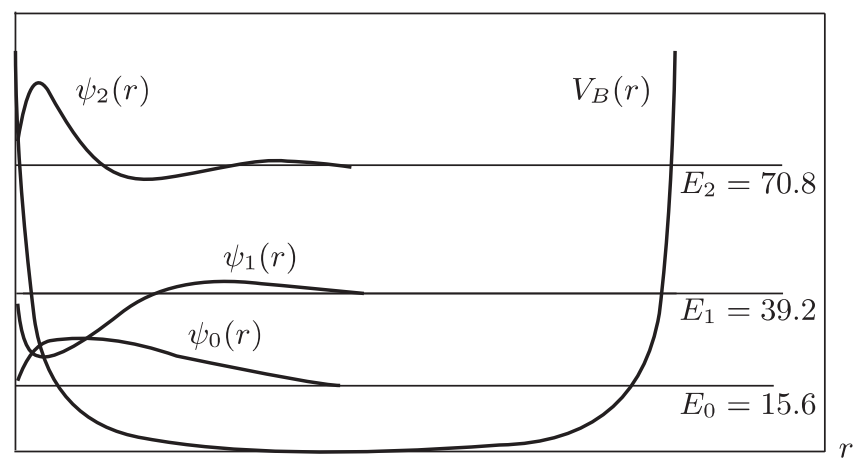

Рис. 3. ТРП в случае $m(r)=e^{g_{B} / b}$ и отвечающие ему собственные значения и собственные функции для $n=0,1,2$.

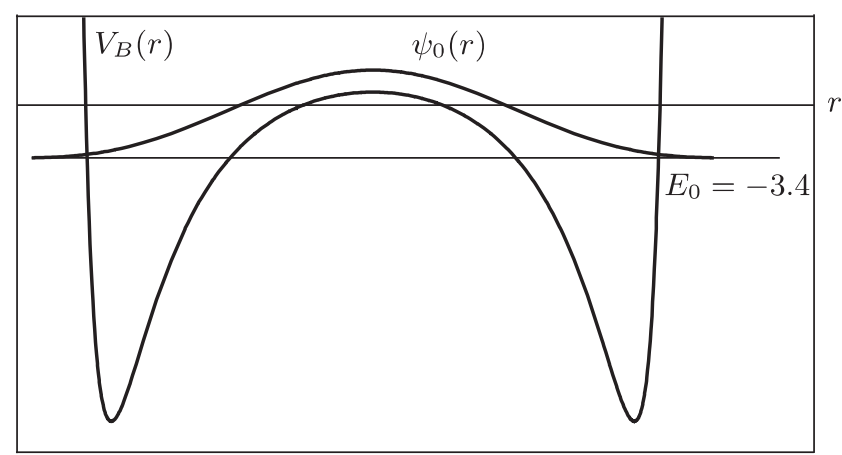

Рис. 4. ТРП в случае $m(r)=\frac{e^{-g_{B} / b}}{1-e^{-g_{B} / b}}$ и вакуумное состояние штурмиана при $\rho_{0}=1, A=2, \epsilon=1.25, C_{B}=1, D=3$. 
модифицированный потенциал

$$
U_{\alpha \beta}(r)=-\frac{s^{2}}{y^{2}(r)-1},
$$

собственные энергии и нормируемые точные собственные функции

$$
\begin{aligned}
E_{n}^{B}= & 4 s^{2}\left[n^{2}+2\left(\epsilon \lambda_{B}+\epsilon n+\lambda_{B} n\right)+\epsilon\right], \\
\psi_{B}(r)= & N_{B} r^{-(D-1) / 2}\left(1-y^{2}(r)\right)^{\epsilon-1 / 2} y^{2 \lambda_{B}+1 / 2}(r) \times \\
& \times{ }_{2} F_{1}\left(-n, 2\left(\epsilon+\lambda_{B}\right)+n ; 1+2 \lambda_{B} ; y^{2}(r)\right)
\end{aligned}
$$

для системы $B$, где $1-s r=y(r), \lambda_{B}=C_{B} / 2 s$,

$$
2 s \epsilon+C_{B}-\sqrt{E_{n}^{B}+4 s^{2} \epsilon(\epsilon-1)+C_{B}^{2}}=-2 s n,
$$

а $C_{B}^{2}$ - характеристическая постоянная (см. рис. 3$)$.

3.1.4. Случай $m(r)=\frac{e^{-g_{B} / b}}{1-e^{-g_{B} / b}}$. В этом случае мы выбираем в качестве рабочего потенциала другой член из $V_{A}(r)$ в $(21)$, т. е.

$$
V_{A}^{\mathrm{w}}(r)=\frac{V_{0} e^{-2 r / b}}{\left(1-e^{-r / b}\right)^{2}},
$$

где

$$
V_{0}=\frac{\epsilon(\epsilon-1)}{b^{2}}, \quad \epsilon \neq 0,1
$$

Из соотношений (13) и (32) получается функция преобразования $g_{B}(r)=2 b \ln \sec \rho_{n} r$, где

$$
\rho_{n}^{2}=\frac{-E_{n}^{B}}{4 \epsilon(\epsilon-1)} .
$$

Функция $g_{B}(r)$ задает функцию массы системы $B$,

$$
m(r)=\operatorname{ctg}^{2} \rho_{n} r
$$

а из формул (14)-(16) и (19) получается штурмиан системы $B$ :

$$
\begin{aligned}
V_{B}(r)= & C_{B}^{2} \operatorname{tg}^{4} \rho_{n} r-4 A \rho_{n}^{2} \operatorname{tg}^{2} \rho_{n} r-\frac{\rho_{n}^{2}}{2} \operatorname{tg}^{2} \rho_{n} r \sec ^{2} \rho_{n} r+ \\
& +\frac{\rho_{n}^{2}}{2} \sec ^{2} \rho_{n} r+\frac{\rho_{n}^{2}}{4} \sec ^{4} \rho_{n} r-\frac{(D-1)(D-3)}{4 r^{2}} \frac{1}{m(r)},
\end{aligned}
$$

где $C_{B}^{2}=-4 b^{2} \rho_{n}^{2} E_{n}^{A}$ есть характеристическая постоянная системы $B$. Остальные характеристики системы $B$ имеют следующий вид: модифицированный потенциал есть

$$
U_{\alpha \beta}(r)=\rho_{n}^{2} \sec ^{2} \rho_{n} r-\rho_{n}^{2} \sec ^{2} \rho_{n} r \operatorname{tg}^{2} \rho_{n} r-\rho_{n}^{2} \sec ^{4} \rho_{n} r,
$$

собственные значения и нормируемые собственные функции энергии суть

$$
\begin{aligned}
E_{n}^{B}= & -C_{B}^{2} \epsilon(\epsilon-1)\left(\frac{A-\epsilon-n(n+2 \epsilon)}{2(\epsilon+n)}\right)^{-2}, \\
\psi_{B}(r)= & N_{B} r^{-(D-1) / 2} \sin ^{2 \epsilon-3 / 2} \rho_{n} r \cos ^{2 \lambda_{B}+3 / 2} \rho_{n} r \times \\
& \times{ }_{2} F_{1}\left(-n, 2\left(\epsilon+\lambda_{B}\right)+n ; 1+2 \lambda_{B} ; \cos ^{2} \rho_{n} r\right),
\end{aligned}
$$

где $\lambda_{B}=C_{B} / 2 \rho_{n}$ и $\epsilon+\lambda_{B}-\sqrt{A-E_{n}^{B} / 4 \rho_{n}^{2}+\lambda_{B}^{2}}=-n$ (см. рис. 4). 
Однако потенциал Штурма системы $B$ в данном случае невозможно привести к нормальному виду.

3.2. Построение ТРП с применением потенциала Морса. ТРП Морса задается выражением [45]

$$
V_{A}(r)=B^{2} e^{-2 \delta r}-B(2 A+\delta) e^{-\delta r} .
$$

Собственные энергии и радиальные волновые функции в случае потенциала Морса имеют вид

$$
\begin{aligned}
E_{n}^{A} & =-(A-n \delta)^{2}, \\
\psi_{A}(r) & =r^{-1} y^{\sigma-n} e^{-y / 2} L_{n}^{2 \sigma-2 n}(y) .
\end{aligned}
$$

Здесь $y=(2 B / \delta) e^{-\delta r}$ и $\sigma=A / \delta$. Мы рассмотрели следующие варианты выбора функций массы (при $k=1$ ).

3.2.1. Случай $m(r)=e^{\delta g_{B}(r)}$. Рабочий потенциал из $(34)$ имеет вид

$$
V_{A}^{\mathrm{w}}(r)=-V_{1} e^{-\delta r},
$$

где $V_{1}=B(2 A+\delta)$. Соотношения (13) и (35) дают

$$
g_{B}(r)=\frac{1}{\delta} \ln \frac{1}{1-\eta_{n} r} .
$$

Для системы $B$ при этом получаются функция массы

$$
m(r)=\frac{1}{1-\eta_{n} r}
$$

и потенциал в форме Штурма

$$
V_{B}(r)=\left(C_{B}^{2}-\eta_{n}^{2}\right) \frac{1}{1-\eta_{n} r}+\frac{\eta_{n}^{2} B^{2}}{\delta^{2}}\left(1-\eta_{n} r\right)-\frac{(D-1)(D-3)}{4 r^{2}} \frac{1}{m(r)},
$$

где

$$
\eta_{n}^{2}=\frac{\delta^{2} E_{n}^{B}}{B(2 A+\delta)}, \quad C_{B}^{2}=-\frac{\eta_{n}^{2} E_{n}^{A}}{\delta^{2}}
$$

$C_{B}^{2}$ - характеристическая постоянная порождаемой системы. Собственные энергии для системы $B$ имеют вид

$$
E_{n}^{B}=\frac{C_{B}^{2} B(2 A+\delta)}{(A-n \delta)^{2}} .
$$

Чтобы привести потенциал $V_{B}(r)$ к нормальному виду, сделаем замену

$$
A \rightarrow A_{n}=\frac{1}{2}\left(\frac{\delta^{2}}{B} \frac{E_{n}^{B}}{\lambda^{2}}-\delta\right)
$$

где $\lambda$ - масштабный множитель. Отсюда $\eta_{n}^{2}=\lambda^{2}=$ const, таким образом, мы получаем нормальный вид потенциала

$$
V_{B}(r)=\left(C_{B}^{2}-\lambda^{2}\right) \frac{1}{1-\lambda r}+\lambda^{2} C^{2}(1-\lambda r)-\frac{(D-1)(D-3)}{4 r^{2}} \frac{1}{m(r)},
$$


модифицированный потенциал

$$
U_{\alpha \beta}(r)=\frac{\lambda^{2}}{4} \frac{1}{(1-\lambda r)},
$$

собственные энергии и нормируемые волновые функции

$$
\begin{aligned}
E_{n}^{B} & =2 C \lambda^{2}\left(\theta+n+\frac{1}{2}\right), \\
\psi_{B}(r) & =N_{B} r^{-(D-1) / 2} z^{\theta}(r) e^{-z(r) / 2} L_{n}^{2 \theta}(z(r)),
\end{aligned}
$$

где $C=B / \delta, \theta=C_{B} / \lambda, z(r)=2 C(1-\lambda r)$.

3.2.2. Случай $m(r)=1-e^{-\delta g_{B}}$. С помощью той же процедуры, что и в предыдущем случае, получаем функцию преобразования

$$
g_{B}(r)=\frac{1}{\delta} \ln \sec ^{2} \eta_{n} r
$$

функцию массы

$$
m(r)=\sin ^{2} \eta_{n} r
$$

потенциал в нормальном виде

$$
\begin{aligned}
V_{B}(r)= & C_{B}^{2} \sec ^{2} \lambda r+4 \lambda^{2} C^{2} \cos ^{2} \lambda r-\frac{\lambda^{2}}{2} \sec ^{2} \lambda r \operatorname{cosec}^{2} \lambda r+ \\
& +\frac{\lambda^{2}}{2} \operatorname{cosec}^{4} \lambda r+\frac{\lambda^{2}}{4} \sec ^{2} \lambda r \operatorname{cosec}^{4} \lambda r-\frac{(D-1)(D-3)}{4 r^{2}} \frac{1}{m(r)},
\end{aligned}
$$

модифицированный потенциал

$$
U_{\alpha \beta}(r)=-\lambda^{2} \operatorname{cosec}^{2} \lambda r\left(\operatorname{ctg}^{2} \lambda r+\operatorname{cosec}^{2} \lambda r\right),
$$

собственные энергии и нормируемые волновые функции

$$
\begin{aligned}
E_{n}^{B} & =8 \lambda^{2} C\left(\theta+n+\frac{1}{2}\right), \\
\psi_{B}(r) & =N_{B} r^{-(D-1) / 2} \sin ^{1 / 2} \lambda r \cos ^{2 \theta+1 / 2} \lambda r e^{-Z(r) / 2} L_{n}^{2 \theta}(Z(r))
\end{aligned}
$$

для системы $B$, где $C=B / \delta, \theta=C_{B} / 2 \lambda, Z(r)=2 C \cos ^{2} \lambda r, \lambda$ - масштабный множитель и $C_{B}^{2}$ - характеристическая постоянная.

3.2.3. Случай $m(r)=e^{-\delta g_{B}}$. Выбирая в качестве рабочего потенциала другой член в выражении для $V_{A}(r)$ из формулы $(34)$,

$$
V_{A}^{\mathrm{w}}(r)=B^{2} e^{-2 \delta r},
$$

получаем

$$
g_{B}(r)=-\frac{2}{\delta} \ln \left(1-\eta_{n} r\right), \quad m(r)=\left(1-\eta_{n} r\right)^{2}
$$


потенциал

$$
\begin{aligned}
V_{B}(r)= & \left(C_{B}^{2}-\frac{\eta_{n}^{2}}{4}\right)\left(1-\eta_{n} r\right)^{-4}- \\
& -4 \eta_{n}^{2} C(2 s+1)\left(1-\eta_{n} r\right)^{-2}-\frac{(D-1)(D-3)}{4 r^{2}} \frac{1}{m(r)},
\end{aligned}
$$

модифицированный потенциал

$$
U_{\alpha \beta}(r)=-2 \eta_{n}^{2}\left(1-\eta_{n} r\right)^{-4},
$$

собственные энергии и волновые функции

$$
\begin{aligned}
E_{n}^{B} & =-C_{B}^{2} C^{2}(s-n)^{-2}, \\
\psi_{B}(r) & =N_{B} r^{-(D-1) / 2}\left(1-\eta_{n} r\right)^{2(s-n)+3 / 2} e^{-Z(r) / 2} L_{n}^{2(s-n)}(Z(r)),
\end{aligned}
$$

где $\eta_{n}^{2}=-E_{n}^{B} / 4 C^{2}, s=A / \delta, C=B / \delta, Z(r)=2 C\left(1-\eta_{n} r\right)^{2}$, а $C_{B}^{2}=-4 \eta_{n}^{2} E_{n}^{A} / \delta^{2}$ есть характеристическая постоянная новой системы. Однако порождаемый таким образом потенциал Штурма (38) невозможно привести к нормальному виду.

3.3. Генерация ТРП с помощью потенциала Халтена. Потенциал Меннинга-Розена превращается в потенциал Халтена при $\epsilon=0$ или $\epsilon=1$. Полагая $\epsilon=0$ в соотношениях (29)-(31), получаем новые ТРП, отвечающие различным функциям масс. Список результатов приведен ниже. Во всех случаях, перечисленных ниже,

$$
\lambda_{B}=\frac{C_{B}}{2 s}, \quad C_{B}-\left(E_{n}^{B}+C_{B}^{2}\right)^{1 / 2}=-2 s n, \quad E_{n}^{B}=4 s^{2}\left(n^{2}+2 \lambda_{B} n\right), \quad y(r)=1-s r .
$$

Эти ТРП получаются, если в качестве порождающей системы выбирается потенциал Халтена.

Случай $m(r)=\operatorname{sech}^{2} s r$ :

$$
\begin{gathered}
V_{B}(r)=C_{B}^{2} \operatorname{sh}^{2} s r+\frac{s^{2}}{4} \operatorname{csch}^{2} s r+\frac{s^{2}}{2}\left(1+\operatorname{cth}^{2} s r\right)-\frac{(D-1)(D-3)}{4 r^{2} m(r)}, \\
U_{\alpha \beta}(r)=-s^{2} \operatorname{ch}^{2} s r, \\
\psi_{B}(r)=N_{B} r^{-(D-1) / 2} \mathrm{th}^{-1 / 2} s r \operatorname{sech}^{2 \lambda_{B}+1} s r_{2} F_{1}\left(-n, 2 \lambda_{B}+n, 1+2 \lambda_{B} ; \operatorname{sech}^{2} s r\right) .
\end{gathered}
$$

Случай $m(r)=1 /\left(1-(s r)^{2}\right)$ :

$$
\begin{gathered}
V_{B}(r)=\left(C_{B}^{2}-s^{2}\right)\left(\frac{1}{(s r)^{2}}-1\right)^{-1}+\frac{3 s^{2}}{4}\left(\frac{1}{(s r)^{2}}-1\right)-\frac{(D-1)(D-3)}{4 r^{2} m(r)}, \\
U_{\alpha \beta}(r)=-\frac{s^{2}}{(s r)^{2}-1}, \\
\psi_{B}(r)=N_{B} r^{-(D-1) / 2}(s r)^{-1 / 2}\left(1-(s r)^{2}\right)^{\lambda_{B}}{ }_{2} F_{1}\left(-n, 2 \lambda_{B}+n, 1+2 \lambda_{B} ;\left(1-(s r)^{2}\right)\right) .
\end{gathered}
$$

Случай $m(r)=1 /\left(1-y^{2}(r)\right)$ :

$$
\begin{gathered}
V_{B}(r)=\left(C_{B}^{2}-\frac{s^{2}}{4}\right)\left(\frac{1}{y^{2}(r)-1}\right)-\frac{(D-1)(D-3)}{4 r^{2} m(r)}, \\
U_{\alpha \beta}(r)=\frac{-s^{2}}{y^{2}(r)-1}, \\
\psi_{B}(r)=N_{B} r^{-(D-1) / 2}\left(1-y^{2}(r)\right)^{-1 / 2} y^{2 \lambda_{B}+1 / 2}(r){ }_{2} F_{1}\left(-n, 2 \lambda_{B}+n, 1+2 \lambda_{B} ; y(r)\right) .
\end{gathered}
$$




\section{4. ЗАКЛЮЧЕНИЕ}

В настоящей работе радиальное уравнение Шредингера с постоянной массой было преобразовано в $D$-мерные МЗК-уравнения Шредингера с помощью метода ОП, что позволило получить новые ТРП (с МЗК). Успешное применение метода зависит от правильного выбора функции массы в терминах функции преобразования $g_{B}(r)$, для которой должно выполняться условие интегрируемости (18). Новые неполиномиальные ТРП с функциями масс были получены с помощью потенциала Меннинга-Розена (см. соотношения (29)-(31), (33)), потенциала Морса (соотношения (36)-(38)) и потенциала Халтена (см. формулы в п. 3.3), которые были выбраны в качестве порождающих систем. Порожденные таким образом потенциалы оказываются по своей природе потенциалами Штурма, большинство из них удается привести к нормальному виду с помощью перегруппировок различных параметров потенциалов. Применяемый метод можно легко обобщить на другие неполиномиальные и полиномиальные потенциалы, порождая тем самым значительное число новых ТРП.

Достаточно просто мы показали, что для систем со сферически-симметричной функцией массы и центральным потенциалом параметры неоднозначности задаются в соответствии с упорядочением Жу-Кремера $\alpha=\gamma=-1 / 2, \beta=0$. Неоднозначности при этом фиксируются и оказываются одними и теми же для всех вновь порождаемых систем.

Благодарности. Авторы глубоко признательны профессору С. А. С. Ахмеду (Gauhati Uriversity, India), под руководством которого была начата эта работа. Его руководство на начальном этапе работы было весьма ценным.

\section{Список литературы}

[1] F. Cooper, A. Khare, U. Sukhatme, Phys. Rep., 251:5-6 (1995), 267-385, arXiv: hep-th/9405029.

[2] S. A. S. Ahmed, Internat. J. Theor. Phys., 36:8 (1997), 1893-1905.

[3] N. Saikia, Turk. J. Phys., 36:2 (2012), 187-196.

[4] Н. Саикиа, С. А. С. Ахмед, 168:2 (2011), 291-298.

[5] S. A.S. Ahmed, B. C. Borah, D. Sharma, Eur. Phys. J. D, 17:1 (2001), 5-11.

[6] S. A. S. Ahmed, L. Buragohain, Phys. Scr., 80:2 (2009), 025004, 6 pp.

[7] S. A.S. Ahmed, L. Buragohain, Eur. J. Theor. Phys., 7:23 (2010), 145-154.

[8] N. Bhagawati, N. Saikia, N. N. Singh, Acta Phys. Polon. B, 44:8 (2013), 1711-1723, arXiv: 1309.3152.

[9] N. Bhagawati, Acta Phys. Polon. B, 45:1 (2014), 15-28, arXiv: 1402.1265.

[10] A. Bharali, Phys. Scr., 88:3 (2013), 035009, 7 pp.

[11] J. P. Srivastava, Elements of Solid State Physics, Prentice Hall of India, New Delhi, 2008.

[12] O. von Roos, Phys. Rev. B, 27:12 (1983), 7547-7552.

[13] R. Sever, C. Tezcan, Ö. Yeşiltaş, M. Bucurgat, Internat. J. Theor. Phys., 47:9 (2008), 2243-2248.

[14] C. Tezcan, Sever R., Ö. Yeşiltaş, Internat. J. Theor. Phys., 47:6 (2008), 1713-1721.

[15] C. Tezcan, R. Sever, J. Math. Chem., 42:3 (2007), 387-395.

[16] S.-H. Dong, M. Lozada-Cassou, Phys. Lett. A, 337:4-6 (2005), 313-320.

[17] B. Bagchi, P. S. Gorain, C. Quesne, Modern Phys. Lett. A, 21:36 (2006), 2703-2708, arXiv: quant-ph/0607122. 
[18] C. Quesne, V. M. Tkachuk, J. Phys. A: Math. Gen., 37:14 (2004), 4267-4281.

[19] M. Jafarpour, B. Ashtari, Adv. Stud. Theor. Phys., 5:1-4 (2011), 131-142.

[20] R. Sever, C. Tezcan, Internat. J. Modern Phys. E, 17:7 (2008), 1327-1334, arXiv: 0712.0268.

[21] S. Meyur, Bulg. J. Phys., 38:4 (2011), 357-363.

[22] B. Roy, P. Roy, Phys. Lett. A, 340:1-4 (2005), 70-73.

[23] A. D. Alhaidari, Phys. Rev. A, 66:4 (2002), 042116, 7 pp.

[24] C. Quesne, SIGMA, 5 (2009), 046, 17 pp.

[25] H. Panahi, Z. Bakhshi, Acta Phys. Polon. B, 41:1 (2010), 11-21.

[26] R. Koç, H. Tütüncüler, Ann. Phys., 12:11-12 (2003), 684-691.

[27] C. Quesne, B. Bagchi, A. Banerjee, V. M. Tkachuk, Bulg. J. Phys., 33:4 (2006), 308-318.

[28] C. Quesne, SIGMA, 3 (2007), 067, 14 pp.

[29] C. Quesne, Ann. Phys. (N. Y.), 321:5 (2006), 1221-1239, arXiv: quant-ph/0508216.

[30] J. Yu, S.-H. Dong, G.-H. Sun, Phys. Lett. A, 322:5-6 (2004), 290-297.

[31] A. G. M. Schmidt, Phys. Lett. A, 353:6 (2006), 459-462.

[32] D. A. Kulikov, V.M. Shapoval, ћ-Expansion for the Schrödinger equation with a position-dependent mass, arXiv: 1206.1666.

[33] A. Schulze-Halberg, Internat. J. Modern Phys. A, 22:8-9 (2007), 1735-1769.

[34] A. Schulze-Halberg, Internat. J. Modern Phys. A, 23:3-4 (2008), 537-546.

[35] G. Bastard, Wave Mechanics Applied to Semiconductor Heterostructure, Les Edition de Physique, Paris, 1988.

[36] M. R. Geller, W. Kohn, Phys. Rev. Lett., 70:20 (1993), 3103-3106.

[37] P. Harrison, Quantum Wells, Wires and Dots. Theoretical and Computational Physics, John Wiley \& Sons, New York, 2000.

[38] L. Serra, E. Lipparini, Europhys. Lett., 40:6 (1997), 667-672.

[39] F. A. de Saavedra, J. Boronat, A. Polls, A. Fabrocini, Phys. Rev. B, 50:6 (1994), 4248-4251, arXiv: cond-mat/9403075.

[40] P. Ring, P. Schuck, The Nuclear Many-Body Problem, Springer, Berlin, 1980.

[41] H. Rajbongshi, N. N. Singh, J. Modern Phys., 4 (2013), 1540-1545.

[42] H. Rajbongshi, N. N. Singh, Acta Phys. Polon. B, 45:8 (2014), 1701-1712.

[43] S. Habib Mazharimousavi, O. Mustafa, SIGMA, 6 (2010), 088, 8 pp.

[44] O. Mustafa, S. H. Mazharimousavi, Internat. J. Theor. Phys., 46:7 (2007), 1786-1796.

[45] J. W. Dabrowska, A. Khare, U.P. Sukhatme, J. Phys. A: Math. Gen., 21:4 (1988), L195-L200. 